\title{
Automatic detection and elimination of periodic pulse shaped interferences in partial discharge measuremel
}

\author{
V. Nagesh \\ B.I. Gururaj
}

Indexing terms: Partial discharge, Interference pulse

\begin{abstract}
The interferences present in partial discharge (PD) measurement can he classified as narrow-band and broad-band, the latter being pulsed shaped. The pulse shaped interferences can be periodic or random with respect to power frequency, the former being very common and strong. The paper describes an algorithm for automatic detection and elimination of periodic pulse shaped interferences in PD measurements. The algorithm is developed on lines similar to that used in decomposing an electromyogram (EMG) into its individual motor unit potentials and is verified with practical and simulated PD measurements. In addition to interference pulse rejection, the algorithm can be used to process data from a digital PD detector for overcoming integration errors, obtaining pulse to pulse time interval histograms and distinguishing different PD pulses based on their waveshapes.
\end{abstract}

\section{Introduction}

The interferences present in partial discharge (PD) measurements can be classified as narrow-band or broadband. The detection of narrow-band interferences is easier, as broad-band PD pulses and narrow-band interferences can easily be differentiated in the frequency domain. The methods for reducing narrowband interferences were discussed in Reference 1 . The broadband interferences are pulse shaped. However, it is not possible to apply directly the frequency domain methods to long record lengths in order to distinguish periodic pulsive interferences from PD and only time domain information can be used. The interference pulses can be periodic with respect to the power frequency cycle, occurring at the same phase angle; for example, pulses arising owing to phase angle controls, commutators of electrical machines and power electronic equipment. The interference pulses can also be random, owing to intermittent switching, stray pickup, lightning etc. From observation of PD pulses over different cycles, it is not possible to detect these random interference pulses as their behaviour is similar to the PD pulses and other methods should be used to detect them.
In order to distinguish between PD and periodic interference pulses, two features can be used. These are the phase distribution and the pulse waveshape. Although PD pulses occur only in certain regions of the power frequency cycle, they do not always occur at the same phase angle in each cycle. The periodic interference pulses occur at almost specific phase angles with a very narrow phase spread. Therefore, the phase distributions for a number of cycles have to be processed to enable recognition of the periodic pulses.

It was observed that in PD measurements the shapes of PD pulses (in solid and gaseous insulation) are very narrow and similar to the impulse response of the PD detector. The power frequency related interference pulses often have larger amplitudes, are generally oscillatory and of longer widths $(>20 \mu \mathrm{s})$. Hence, the pulse shape can also be used as a distinguishing feature.

The methods proposed earlier for eliminating periodic external interferences can be classified as analogue and digital. The analogue methods used the bridge detection method, the reference signal method or the current and voltage direction of the pulses to detect and eliminate external interferences. Hilder and Black [2] have suggested a method which uses a combination of bridge detection and reference signal methods for PD measurements in cables. The bridge method uses the principle that the direction of the external interference pulses is the same in both arms of the bridge, whereas PD pulses from the test object pass through the input units in the opposite direction. This helps in detecting internal discharges. To avoid certain types of radiated interferences, an external reference signal from an aerial is used. When strong pulses are detected, the output of the PD detector is gated to avoid these large pulses appearing in the PD display. Some other commercially available detectors also use this method.

Borsi and Hartji [3] have proposed a method which uses a modified Rogowski coil to discriminate between internal PD pulses and external interference. It uses the relative directions between the voltages produced by the pulse in the Rogowski coil to enhance or suppress the pulse. The method is efficientin suppressing certain types of conducted external interferences. The performance of this method against pulses that get coupled by radiation, having similar voltage directions, is not reported.

Digital methods based on digital signal processing (DSP)using the phase information has been proposed by Okamoto and Tanaka [4] and Konig et al. [5]. In Reference 4 the distribution of the number of pulses at different phase angles is plotted. The strongly periodic pulses appear as sharp peaks and are automatically eliminated. 
A similar method is used in Reference 5 to extract the periodic interference pulses from the randomly varying PD pulses. The data is recorded over a number of cycles. The data for each cycle is then averaged with the data of consecutive cycles at the same phase angles. To avoid the effect of jitter in the pulses, care is taken to see that the position in each cycle corresponds to the same phase angle. This averaged record is used as the template to locate and subtract the prominant periodic pulses. The method gives good results when PD pulses are less in number and well distributed, but will lead to ambiguity if the number of pulses are large and strongly persistent.

In an alternate approach suggested by Konig et al. [5] records made without voltage application are taken to get an unambiguous interference pulse template. Such a method can be used if the test object is the only load fed by the high voltage source and cannot be applied to continuously energised equipment.

From this survey, it is seen that different approaches for detection and elimination of periodic interference pulses have been used. The major drawback of analogue methods is their inefficiency in rejecting radio emissions, especially if many of such emissions are present. A digital method which is fast and efficient in rejecting such emissions with minimum distortion to all PD and interference pulses is reported in Reference 1. After the removal of the radio emissions the same record can be processed further to reject external interference pulses.

The waveshape of the PD pulses is different from these periodic interference pulses and this difference can also be used for detecting periodic interferences. However, its use has not yet been reported in the published literature. For detecting and rejecting periodic interferences, a single channel record is adequate. An algorithm has been developed which identifies individual pulses and then clusters all the pulses which have similar waveshapes, so that their individual pulse-phase distributions can be studied. This helps in correctly identifying whether the cluster(s) is $\mathrm{PD}$ or interference. If the cluster consists of interference pulses then it is deleted, and the remaining clusters which are predominantly of PD only are used to reconstruct the record. The details of the algorithm and its performance with practical data is discussed in this paper.

\section{Possibilitiesof adaptating biomedical signal processingtechniques}

The clustering of pulses based on waveshape and time of occurrence is well established in biomedical signal processing, as applied to an electromyogram (EMG). An EMG is the signal obtained by measuring the electrical activity in a muscle. The muscle fibres are activated by neurons located in the spinal cord. One single neuron stimulates a muscle fibre group (motor unit, MU) that radiates a specific waveform called a motor unit action potential (MUP) as shown in Fig. 1A. The repeated innervation of several individual motor units results in a superposition of pulse trains, which constitute the EMG [6]. The parameters of MUP waveshapes have proven to be of importance for clinical diagnosis. Hence, EMG is decomposed into individual MUPs and its firing statistics are obtained. The PD process resembles the EMG in a normalised scale and the techniques used for decomposing the EMG can also be applied to PD and interference (Fig. 1B). The methods suggested by Gerber et al. [6] and McGill et al. [7] were used for decomposing PD and noise, with suitable modifications (described at appropri- ate places). The detailed description of each stage of the processing is discussed below.

\subsection{Data acquisition}

The conventional straight detection method of PD measurement was used for measurement in an unshielded

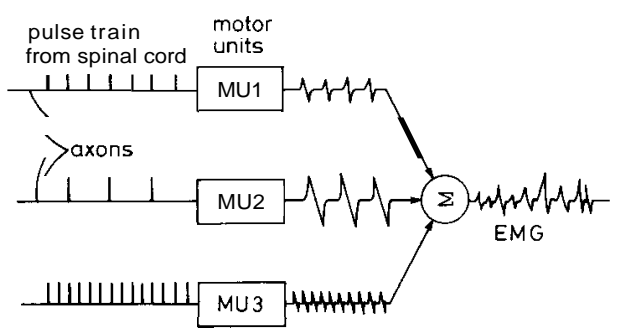

Fig. 1A Physiological process involved in generation of EMG

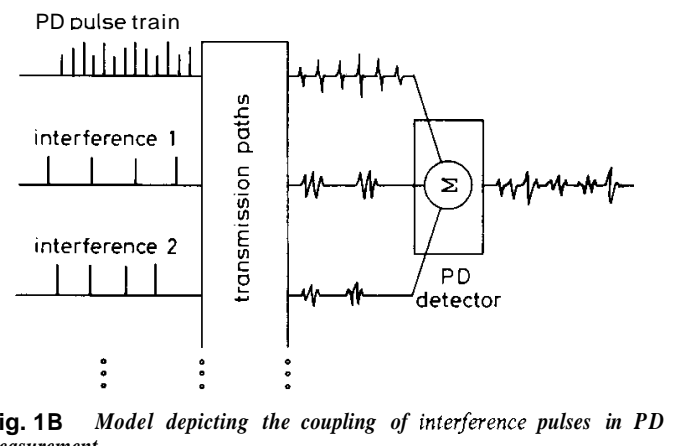

measurement

environment. The output of the PD detector was fed into a ten bit digitiser (Tektronix RTD 710A) and the digitised data was processed using a PC. The sampling rate to be used for recording depended on the bandwidth of the PD detector and coupling unit. The bandwidth of the PD detector chosen is usually a compromise between PD pulse resolution and the quasi-integration of the pulse. It was found that a sampling time of $1 \mu \mathrm{s}$ for a PD detector bandwidth of $40 \mathrm{kHz}$, and $0.5 \mu \mathrm{s}$ for a bandwidth of $250 \mathrm{kHz}$ was sufficient. A record length of 256000 samples was chosen, so that the record extended over at least five power frequency cycles.

\subsection{Decomposition of noisy input record}

The procedure for decomposition is briefly described in the flow chart shown in Fig. 2. To remove the presence of any discrete spectral interferences (DSIs) such as radio emissions, prefiltering has to be effected. The prefiltering is performed using the method suggested in Reference 1 . After the removal of DSIs the pulses become visible and can be identified more easily.

\subsection{Segmentation of pulses}

In order to classify the pulses according to their waveshapes, individual pulses must be recognized. For this, pulse boundaries must be automatically identified. Different pulse segmentation methods are reported in EMG and ECG literature. A segmentation method which determines the pulse boundaries using the average power over a given window is reported in Reference 6 . In an alternative method [7], the data is passed through a high-pass 
filter to enhance the spikes. When these values exceed a preset threshold, a pulse is said to be detected. The boundaries of the pulses can also be identified by using the instantaneous curvature, as reported in Reference 8

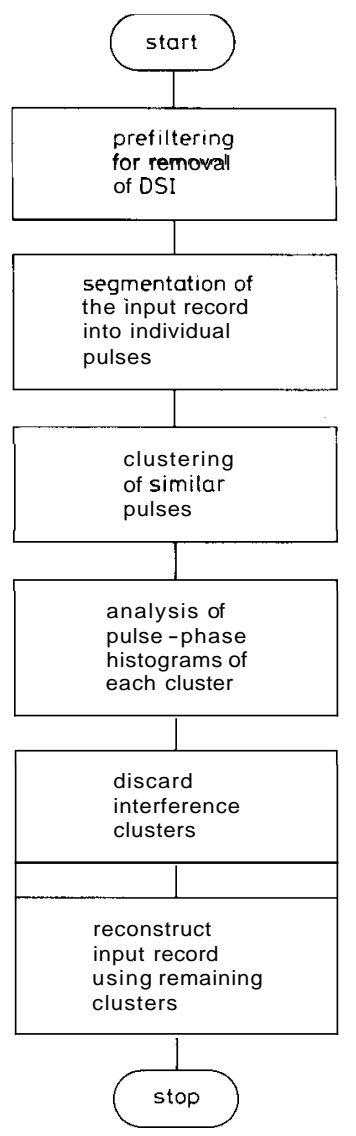

Fig. 2 Stages involved in detection and elimination of interferences

for ECG records. It was found that using any one of these methods alone is not efficient in detecting both the fast PD pulses and the wider periodic pulsive interferences. Hence, a combination of these methods was used in the algorithm.

The procedure adopted for detecting the pulses and identifying their boundaries is shown in Fig. 3. If the instantaneous height is greater than a preset threshold, then the pulse is said to be detected. The threshold is chosen to be about five times the noise floor level. As some pulses oscillate before decaying, the pulse height itself cannot be used for identifying the end of pulse. The average power given by the following equation:

$$
P[i]=\sum_{k=-N}^{k=+N}(x[i+k]-p)^{2} \times a
$$

where

$$
\begin{aligned}
p & =2 \times \text { noise floor level } \\
\mathrm{a} & =0 \text { if } x[i]<\mathrm{p} \\
& =1 \text { if } x[i]>\mathrm{p} \\
\mathbf{2 N} & =\text { length of window use for averaging }
\end{aligned}
$$

as well as the approximate instantaneous curvature:

$$
C[i]=x[i-2]-2 x[i]+x[i+2]
$$

are used for identifying the end of the pulses.

Proper identification of the pulses is necessary as the full pulse should should be captured; more so, if two

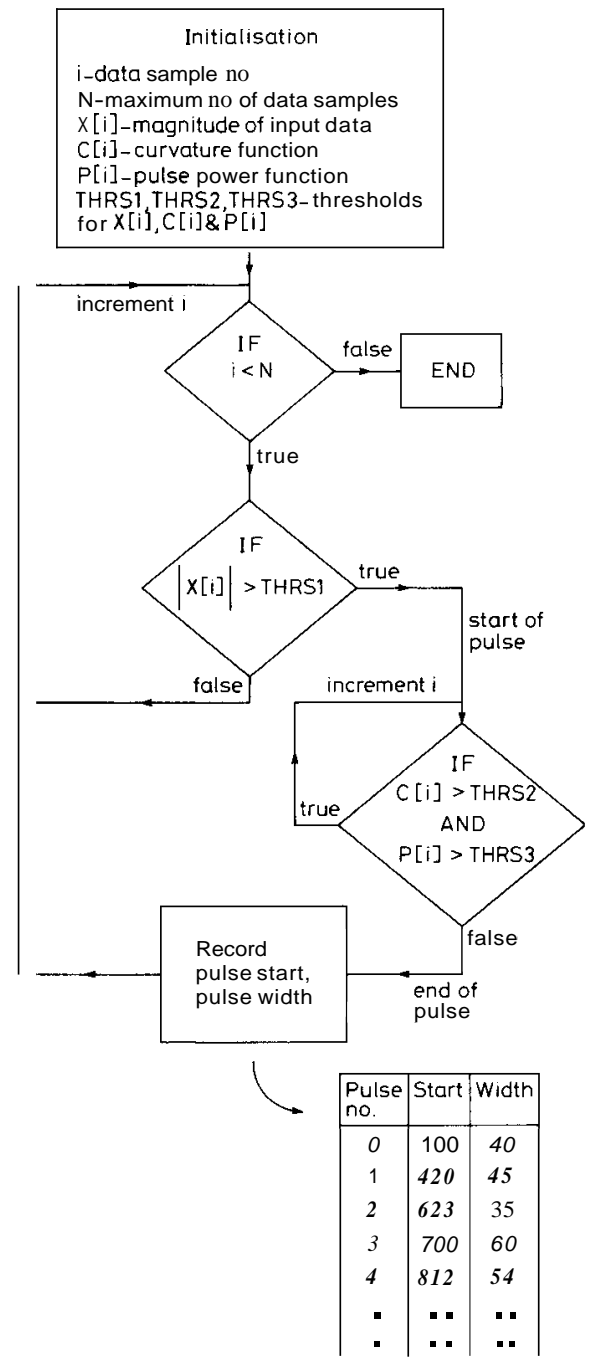

pulses occur near each other. The thresholds were chosen by trial and error. If a large threshold value is chosen, then only pulses which are large and narrow get detected. If the threshold is small, then the tail end portion is well recognised, but if two pulses occur near each other then it may fail to separate them and both the pulses will be treated as a single pulse. In EMG records, the pulses that are generally encountered are very sharp and pulse detection is easier and some methods for deriving these thresholds have been proposed. However, in PD measurements, the PD pulses are sharp, while the interference pulses are of longer duration, varying over different lengths. Hence, a trial and error method has to be adopted. However, these thresholds can be set as constants, as long as the sampling rate and the bandwidth of the detector are not 
changed. For accurate pulse detection, the noise floor level should be small. Pulses of smaller width are more correctly detected when compared with slowly decaying oscillatory pulses.

Using the above method, a table listing the pulse number, its starting position and its width is created as shown in Fig. 3. This table is used for clustering the pulses. Fig. 4 shows the segmentation of a small portion of the record using the above method.

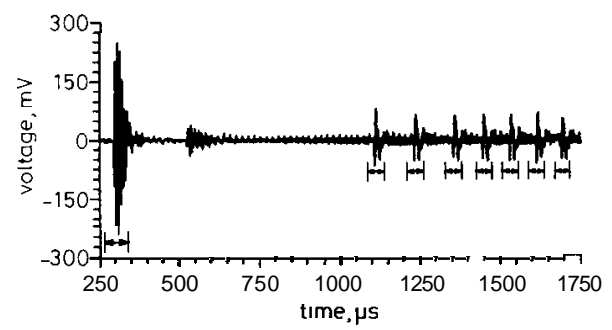

Fig. 4 Record showing the segmentation of pulses

\subsection{Clustering similar pulses}

Once the pulses are segmented, a clustering algorithm is used to group similar pulses so that the pulse-phase angle distributions of each cluster can be separately identified to detect interference pulse clusters. The clustering of similar pulses is done by cross-correlation of individual pulses. The cross-correlation can be done either in the frequency domain or in time domain. The frequency domain method is popular in EMG decomposition [6], it was also found to be convenient in decomposing noisy PD measurements, as only the magnitude spectrum of the pulse is used for cross-correlation instead of the actual time domain pulse. This avoids the need for the alignment of the two pulses before cross-correlating them. Since the pulse lengths are small ( $<128$ samples) FFT computation is faster. The length of the pulses are made up by padding zeros to a total of $n=64$ or 128 . The flow chart used for clustering is described briefly in Fig. 5. If the FFT magnitude of the pulses ( $\boldsymbol{i}$ and $\mathbf{j}$ ) to be compared are $F_{i}, F_{j}$, the cross-correlation $\mathbf{C C}$ defined by

$$
C C=\frac{\sum_{k=1}^{k=n / 2} F_{i}[k] \times F_{j}[k]}{\sum_{k=1}^{k=n / 2} F_{i}[k]^{2} \times \sum_{k=1}^{k=n / 2} F_{j}[k]^{2}}
$$

is calculated for each pulse pair, as long as they are not identified with any clusters (selected). If the pulses are similar they are put in the same cluster and a cluster table is created.

In EMG methods [6, 7], clustering is achieved in two passes, the first pass is similar to that described in Fig. 5; in the second pass the template of the pulse waveshape is formed for each cluster by averaging the pulse waveshapes. This mean waveshape is used as a representative for the given cluster. The second pass of preparing the template was found not to be necessary in the case of $\mathrm{PD}$, as it is the pulse-phase information rather than the exact pulse shape of the cluster which is interesting.

After the clusters have been identified, the pulse-phase histograms are generated for each cluster as shown in Figs. 6C and 6D, for the signal shown in Fig. 6A. A record length of six power frequency cycles sampled at $1 \mu \mathrm{s}$ was used for a point to plane configuration $(5 \mathrm{~cm}$ gap) at $20 \mathrm{kV}$. In all, 254 pulses were detected, 252 pulses were classified and the remaining two did not fit into any cluster and were discarded as stray noise.

The algorithm then detected clusters which had strongly periodic discrete peaks. It identified clusters $3, \mathbf{4}$, 5 as periodic and they were classified as interference. On close observation of the record, the classification was

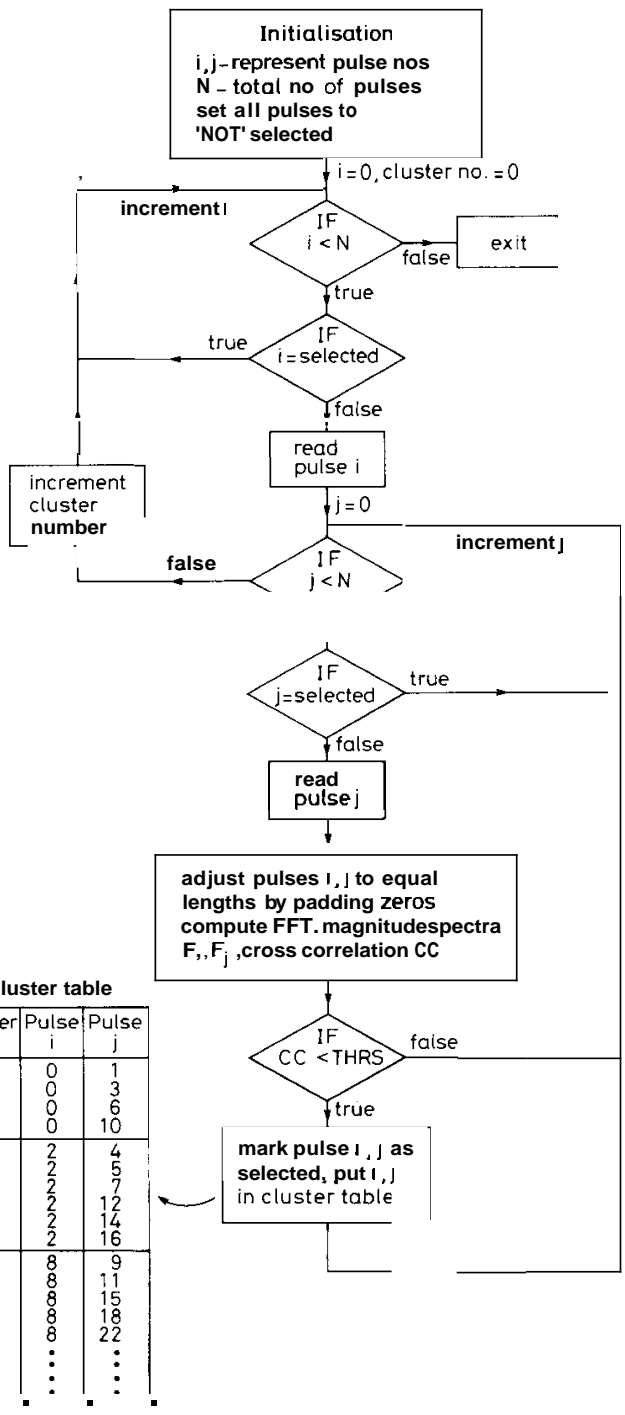

Fig. 5 Procedure usedfor clustering of pulses

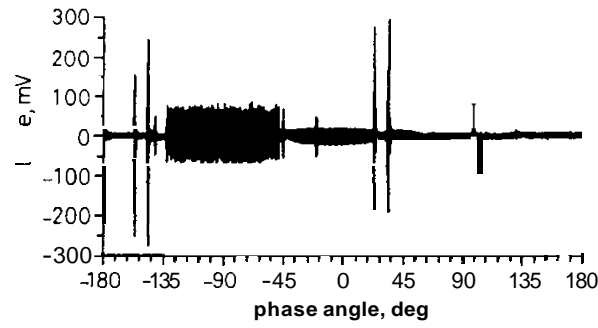

Fig. 6A Plot of one cycle of input record obtained using a point to plane configuration 
found to be correct. The interference pulse clusters were then discarded and the record consisting only of the PD was reconstructed using the remaining clusters as shown in Fig. 6B. It was found that time interval between cycles

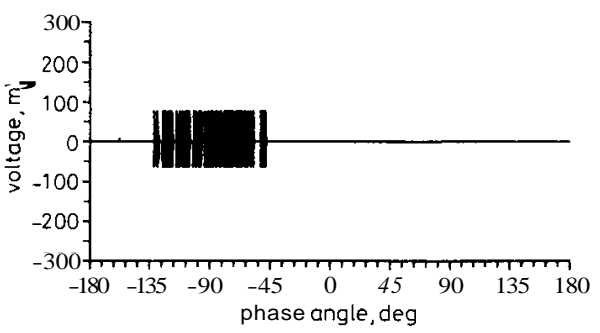

Fig. 68 Plot ofreconstructed outputfor the input shown in Fig. 6 A

need not be known exactly; a value within $+60 \mu$ s was found to be sufficient.

The performance of the above method was also tested with records containing the internal PD of a transformer at $20 \mathrm{kV}$. At the time of recording the records did not contain periodic interference pulses. In order to test the performance of the above method, periodic interference pulses recorded without the application of voltage were
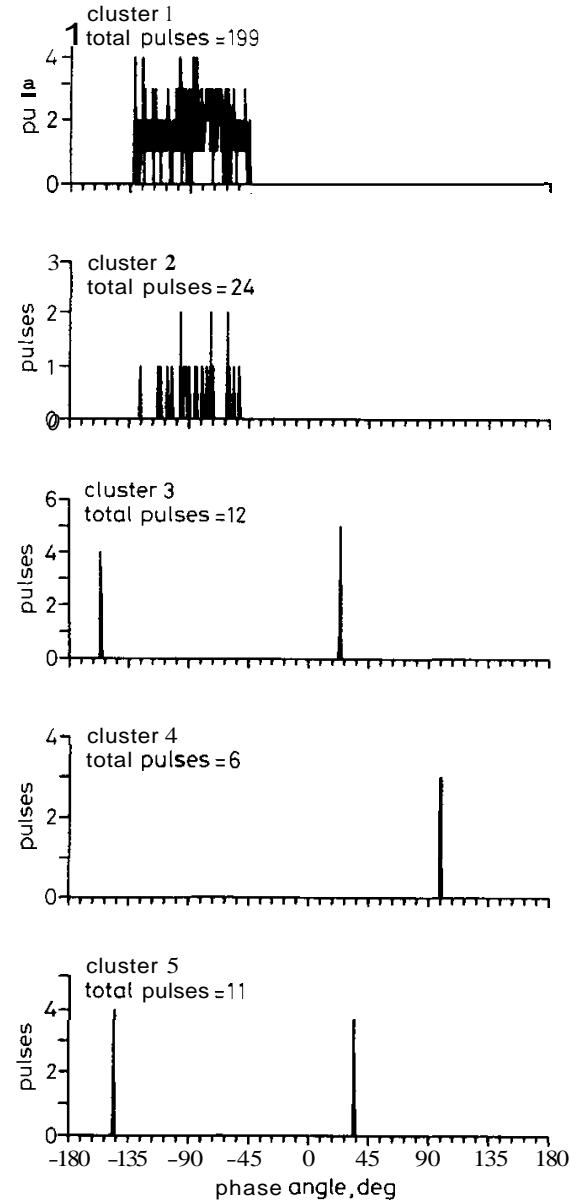

Fig. 6C Pulse-phase diagrams of the clusters superposed on the actual PD record to simulate interference.

A record length of six cycles was used and, in all, 142 pulses were detected. These were classified into nine clusters. Fig. 7, shows the actual record with some of the
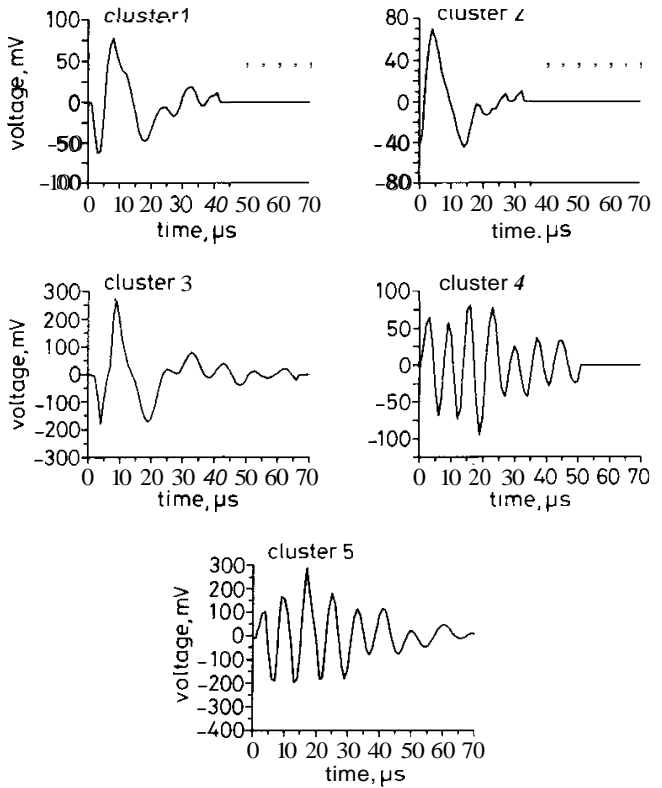

Fig. 6D Pulses representing their corresponding clusters

clusters and their phase distributions and the reconstructed record without the periodic interference pulses. Cluster 0 represents the actual PD in the transformer itself and clusters 4,5 and 6 represent periodic interferences. The oscillatory pulse in cluster 5 is the same as the pulse in cluster 6, except that segmentation was not possible when the PD pulse occurred very near the inter-

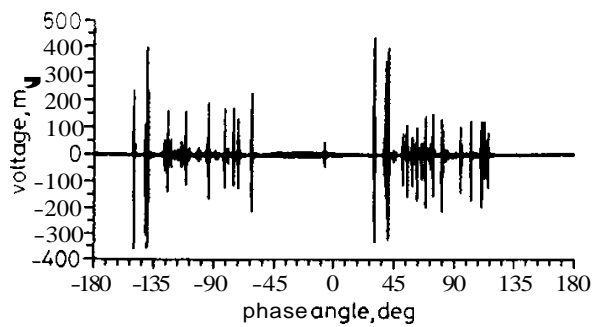

Fig. 7A Plot of one cycle of input record of internal PD in transformers

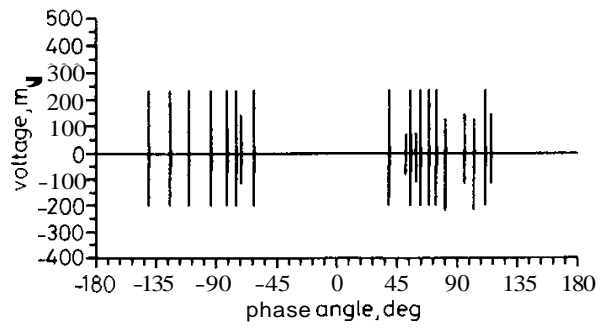

Fig. 78 Plot ofreconstructed output for the input shown in Fig. $7 \mathrm{~A}$ 
ference pulse, hence it was put into a separate cluster. All the interference pulses were detected as in the earlier example.
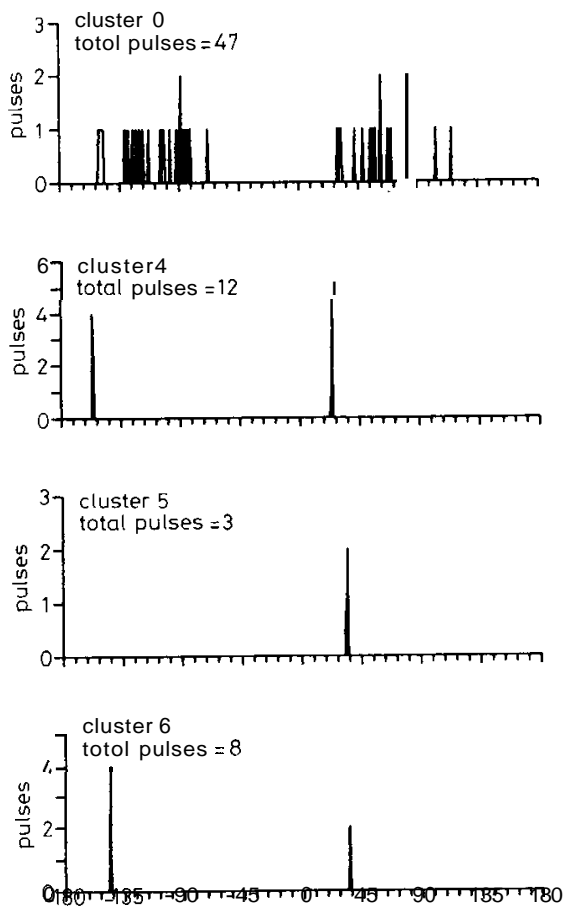

phase angle, deg

Fig. 7C Pulse-phase diagrams of some ofthe clusters
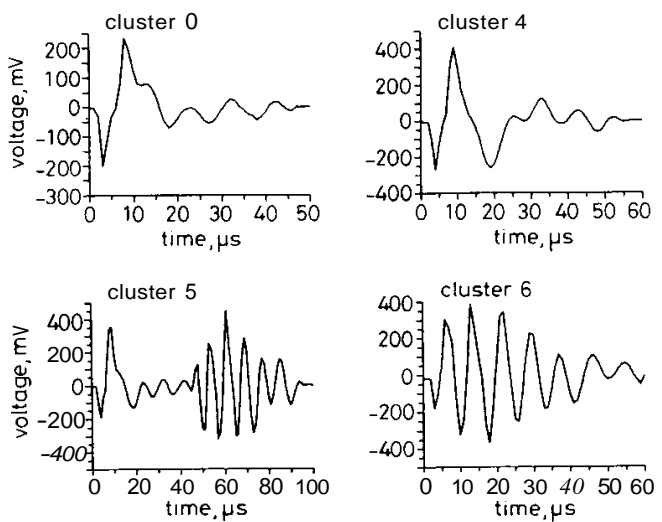

Fig. 7D Pulses representing their corresponding clusters

To study the behaviour of the pulses when they occur very near to each other, or when the pulses overlap, a record containing corona pulses generated by sharp wire at $90 \mathrm{kV}$ was used. A burst of pulses with a repetition time of less than $40 \mu$ s were recorded over a length of five cycles with a sampling time of $500 \mathrm{~ns}$ (PD detector and input unit bandwidth $=250 \mathrm{kHz}$ ). Fig. 8 shows the record for one cycle. In all, 497 pulses were detected. The shape and pulse-phase histograms are shown for a few clusters. The clusters 0 and 1 are clearly due to corona. Clusters 4, 8 and 10 also represent corona, but here the pulses have overlapped, the segmentation algorithm was unable to distinguish between two pulses when they occurred within $15 \mu \mathrm{s}$. The algorithm considered such overlapping pulses as separate and put them into new clusters. Cluster 9 was detected as periodic.

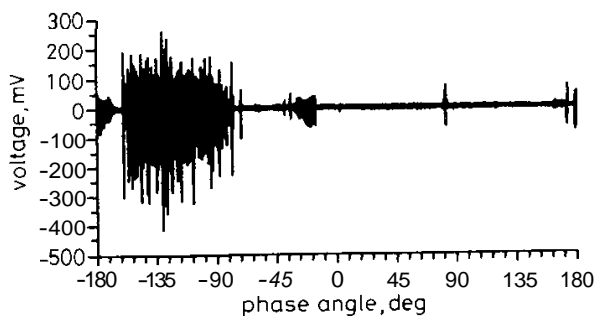

Fig. 8A Plot of one cycle of input record of corona from a sharp point at $88 \mathrm{kV}$
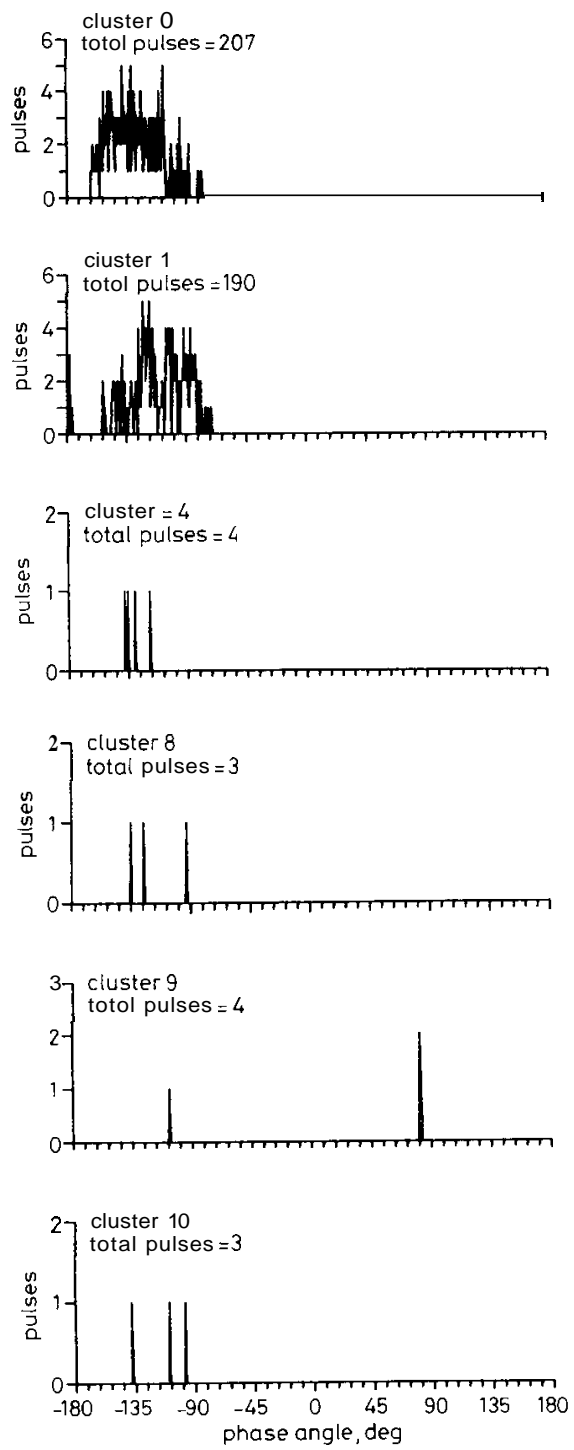

Fig. 8B Pulse-phase diagrams of some ofthe clusters 
The times involved in processing are variable. The segmentation time is nearly constant, but the clustering time varies with the number of pulses. For processing the record (on an IBM PC386 at 16 MHz) shown in Fig. 6 , the segmentation time was $25 \mathrm{~s}$ and the clustering time was $30 \mathrm{~s}$.
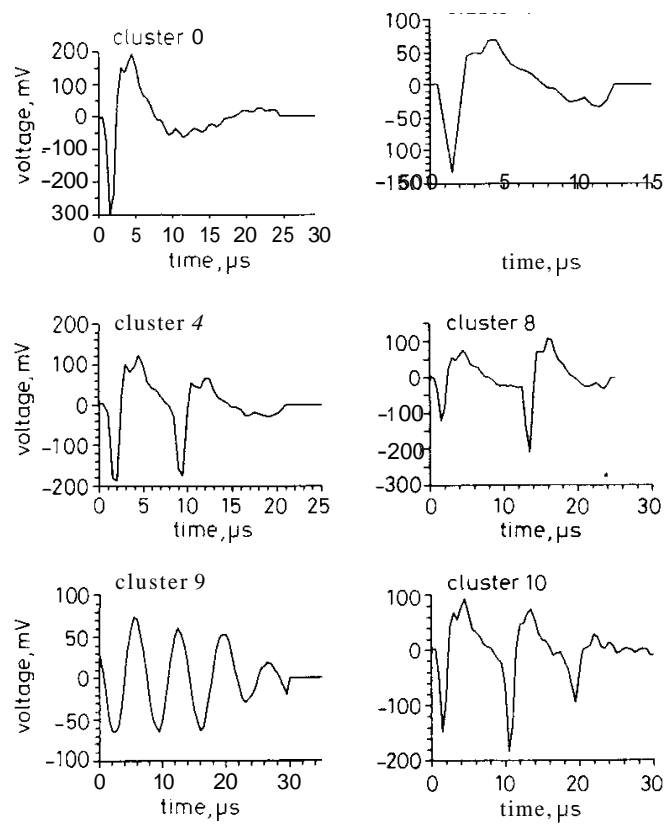

Fig. 8C Pulses representing their corresponding clusters

\section{Other applications of the method}

In addition to the elimination of periodic interferences, the method also gives the pulse time intervals, for example, Fig. 9 shows the histograms of the pulse to pulse time intervals for the corona $(90 \mathrm{kV})$ and the internal PD in the transformer $(20 \mathrm{kV})$. It can be seen that the distributions are different. At present, PD pattern recognition mainly uses the phase distributions; the pulse to pulse time histograms also give more insight into the process and can also be used along with the phase information.
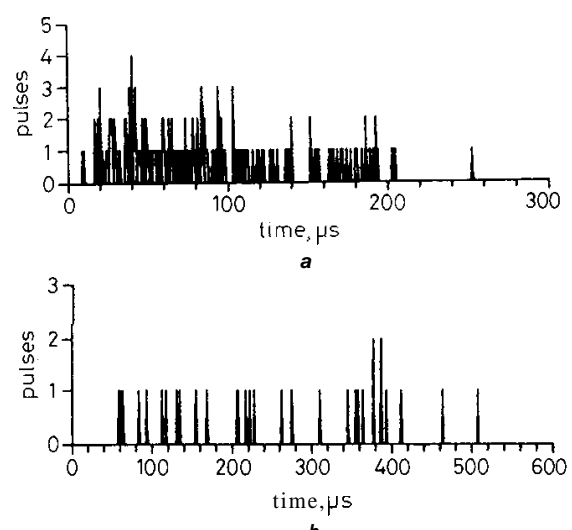

Fig. 9 Histograms of pulse to pulse time intervals

a corona $(88 \mathrm{kV}$

$b$ internal PD of transformer $(20 \mathrm{kV})$
While measuring long duration PD pulses in liquid insulation, integration errors will arise. The segmentation algorithm can be used to identify the boundaries of each of these PD pulses, so that deconvolution methods can be applied on the individual pulses to obtain the correct charge.

In the case of rotating machines and transformers which have distributed structures, the PD pulses originating from different points will have different shapes. If the bandwidth of the PD detector is sufficiently high, then the above clustering algorithm can be used to decompose the multiple PD source record into individual PD processes which can be processed further to get more information on the PD location.

The pulse segmentation algorithm can also be applied to the PD in a cable to compute the pulse to pulse time intervals, which will be helpful in locating the position of the PD source.

\section{Conclusions}

Periodic pulse shaped interferences are very common in PD measurements and always occur at specific phase angles when compared with PD pulses. In addition, the shapes of PD pulses and periodic interference pulses are different. An algorithm was developed which uses the phase distribution and pulse shape to detect and eliminate periodic pulses, using DSP techniques. The algorithm identifies individual pulses and then clusters all the pulses which have a similar shape, so that their individual phase distributions can be studied. This helps in correctly identifying whether the cluster(s) is PD or interference. The techniques used in decomposing an electromyogram (EMG) into individual motor unit potentials has been used with suitable modifications for detection and clustering of pulses in PD measurements. If the cluster consists of interference pulses then it is deleted and only the remaining clusters which are predominantly of $\mathrm{PD}$ are used to reconstruct the record.

The algorithm was applied to practical data records of corona at low and high voltages. In addition, periodic interference pulses were superposed on a record contain ing the internal PD of transformers. The algorithm correctly identified the interference pulses and eliminated them. It was only when pulses occurred very near to each other that the algorithm failed to separate them and considered both of them as a single pulse. If the pulses are large in number, the clustering of all the pulses takes a longer time, this drawback can be overcome by using faster or parallel processors.

The above algorithm has other applications. It can be used to study pulse to pulse time distributions and also obtain statistics of the PD process, these are useful for $\mathrm{PD}$ pattern recognition, ageing studies and locating PD in cables.

It is also possible to integrate deconvolution methods with this algorithm to compute the exact charge of longer duration PD pulses. The clustering algorithm can be used to distinguish different sources of PD in transformers and rotating machines, as the pulse shapes change with location. With these applications, it can be used as a general purpose algorithm to process data from a digital PD detector.

\section{References}

1 NAGESH, V., and GURURAJ, B.I.: 'Evaluation of digital filters for rejecting discrete spectral interference in on-site PD measurements', IEEE Trans., 1993, EI-28,(1), pp. 73-85 
2 HILDER, D.A., and BLACK, I.A.: 'Noise suppression methods for partial discharge measurements on single and three-phase cables'. 5th International conference on dielectric materials, measurements and applications, Canterbury, UK, 27th-30th June 1988, IEE Publication No. 289

3 BORSI, H., and HARTJE, M.: 'Application of Rogowski coils for partial discharge (PD) decoupling and noise suppression'. 5th International Symposium on High Voltage Engineering, Braunschweig, Federal Republic of Germany, 24th-28th August 1987, paper No. 42.02

4 OKAMOTO, T., and TANAKA, T.: 'Novel partial discharge measurement computer-aided measuring system'; IEEE Trans.. 1986, EI-21,(6), pp. 1015-1019

5 KONIG, G., KOPF, U., and FESER, K.: 'Methods to improve on-site partial discharge measuring instruments'. 7th International Svmoosium on High Voltage Engineering, Technische Universitat Dresden, 26th-30th August 1991, paper No.73.02

6 GERBER, A., STUDER, R.M., DE FIGUEIREDO, R.J.P., and MOSCHYTZ. G.S.: 'A new framework and computer program for quantitative EMG signal analysis', IEEE Trans., 1984, BME-31, (12), pp. $857-863$

7 MCGILL, K.C., CUMMINS, K.L., and DORFMAN, L.J.: 'Automatic decomposition of clinical electromyogram',IEEE Trans., 1985 ,

BME-32, (7), pp. 470-477
8 JAGER, F., KOREN, I., and GYERGYEK, L.: 'Multiresolution representation and analysis of ECG waveforms'. IEEE 1990: Conference proceedings of Computers in Cardiology, pp. 547-500 